\title{
Paul Hamilton Wood (1907-1962). El máximo exponente de la cardiología clínica Británica del siglo XX
}

\author{
JUAN D. HUMPHREYS ${ }^{1}$, PABLO YOUNG ${ }^{2}$
}

\section{Paul Hamilton Wood. The foremost British clinical cardiologist of the 20th century}

In the United Kingdom, during the mid-20th century, Paul Wood appears as the new leader of European cardiology. He introduced rigorous bed-side diagnostic methods and the confirmation of these clinical findings by cardiac catheterization, in an effort to demonstrate the pathophysiological causes of cardiac disease. In his search for the correct diagnosis, his comments, which could be caustic, both impressed and offended many. He had a strong commanding personality and was intensely honest in his appreciations. His showmanship and diagnostic ability became renown. In 1950, the publishing of the first edition of his textbook "Diseases of the Heart and Circulation" brought him worldwide recognition. In this book, Wood introduces his personal fresh style of narrative and his physiologic approach to cardiology. His intense professional activity, teaching, lecturing and preparing the third edition of his book, plus the fact that he was a heavy smoker, must have been the factors that lead to a myocardial infarction and death at the early age of 54. As Paul Dudley White and Ignacio Chávez in America, Paul Wood in Europe will be remembered as the emblematic figure leading the transition of cardiology into the modern era.

(Rev Med Chile 2012; 140: 121-124).

Key words: Cardiology; Diagnosis, differential; History of Medicine.
Servicios de ${ }^{1}$ Cardiología y ${ }^{2}$ Clínica Médica, Hospital Británico de Buenos Aires, Argentina.

Universidad Católica Argentina.

Recibido el 15 de junio de 2011, aceptado el 22 de agosto de 2011

Correspondencia a: Pablo Young, Hospital Británico. Perdriel 74 (1280) Buenos Aires, Argentina. Tel 541143096400 Fax 541143043393 E-mail: pabloyoung2003@ yahoo.com.ar

\section{Perspectiva histórica}

A mitad del siglo pasado, mientras Paul Dudley White (1886-1973), en los Estados Unidos de Norteamérica e Ignacio Chávez Sánchez (1897-1979) en Méjico impulsaban el desarrollo de la cardiología moderna en América; en el Reino Unido surge la figura de Paul Wood (Figura 1) liderando la respuesta europea. Estos tres emblemáticos cardiólogos constituyen las principales figuras de la transición de la vieja cardiología a la era moderna.

Wood fue un clínico brillante e inspirado. Tal vez su mayor contribución a la medicina fue el refinamiento y la perfección de su capacidad diagnóstica al lado de la cama del paciente. Introdujo el concepto cuantitativo en el registro de los datos clínicos. Abordaba al paciente nuevo estableciendo hipótesis diagnósticas, y luego realizaba el interrogatorio y un examen físico minucioso a los fines de probar sus suposiciones. Utilizando esta metodología iba pensando su diagnóstico formulando nuevas hipótesis y recabando nueva información clínica con absoluto rigor científico. A través de este proceso iterativo de prueba y contra prueba, aprobando y descartando las diversas posibilidades llegaba a un diagnóstico de trabajo. Este diagnóstico luego era ratificado o rectificado sometiendo al paciente a un cateterismo cardiaco que Wood en algún momento definió como el "patrón oro". Dado que una gran cantidad de los pacientes padecían de enfermedad reumática o de cardiopatías congénitas, este enfoque habitualmente permitía dilucidar la fisiopatología del proceso y llegar a un diagnóstico final. De esta manera introdujo precisión en la evaluación preoperatoria de los pa- 


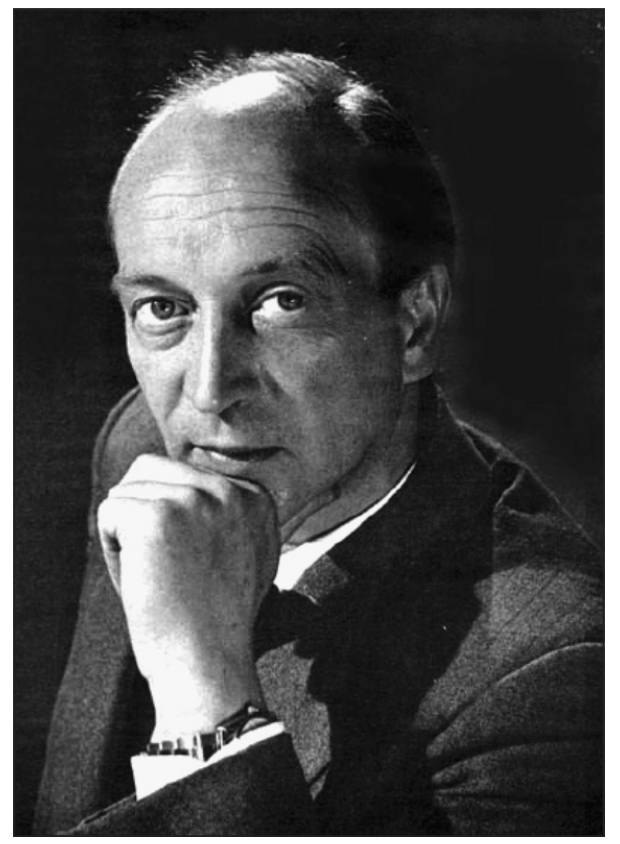

Figura 1. Dr. Paul Hamilton Wood. Tomado de una fotografía publicada en Br Heart J 1962; 24: 661 ${ }^{1}$

cientes con cardiopatías congénitas y reumáticas, con o sin hipertensión pulmonar ${ }^{1-3}$.

\section{Su vida y estudios}

La carrera de Paul Wood fue meteórica. Nació el 16 de agosto de 1907 en Coonoor, Tamil Nadu, India donde su padre trabajaba como empleado civil del Gobierno Colonial Británico. Siguiendo la dura tradición británica, a la edad de tres años y medio fue enviado al colegio en Inglaterra como pupilo. Allí permaneció hasta los trece años en que pasó a Tasmania, Australia adonde sus padres habían emigrado para dedicarse al cultivo de frutales. Paul completó sus estudios secundarios en Tasmania, para luego pasar al Trinity College de la Universidad de Melbourne, donde en 1931 se graduó en Medicina y Cirugía siguiendo los pasos de su abuelo y bisabuelo. Fue un estudiante desparejo: aprobó con honores cirugía y obstetricia, pero reprobó su primer examen de medicina y tuvo que darlo nuevamente. Por este motivo quedó relegado a realizar su internado en Christchurch, Nueva Zelanda. Allí conoció a Elizabeth Guthrie con quien se casó en 1934 viviendo ya en Londres. De esta unión nacieron sus tres hijos, dos varones y una mujer. Fue un entusiasta jugador de fútbol y de rugby, representando a su universidad en diversos encuentros, e integró el equipo universitario australiano contra los equipos universitarios de Japón y Nueva Zelanda. También se destacó en tenis y en el ski de fondo. Era muy jovial y afecto a las típicas travesuras estudiantiles de la época ${ }^{2,4}$.

En 1933 Wood retorna al Reino Unido con el objetivo de especializarse. Aprueba el examen de ingreso como Miembro del Colegio Real de Médicos (MRCP) y acepta un nombramiento en el Brompton Hospital ${ }^{4}$. El año siguiente, a la edad de 27 años, obtiene el puesto de Residente en el National Heart Hospital de Londres. En este puesto es reconocido por la profundidad de sus conocimientos y su excepcional juicio clínico ${ }^{2}$. El año siguiente la Universidad de Londres decide fundar la Escuela Real de Medicina de Postgrado y el Hammersmith Hospital fue designado como sede clínica. El Profesor de Medicina Sir Francis Fraser (1885-1964) invita a Wood a organizar la sección de Cardiología. Allí enseña a estudiantes y comienza a realizar investigación clínica. En 1938 es nombrado médico de consultorios externos del National Heart Hospital y Consultor de Hammersmith Hospital ${ }^{2,4}$. En 1940 es nombrado Fellow del Colegio Real de Médicos (FRCP) y el año siguiente recibe el Doctorado en Medicina de la Universidad de Melbourne (MD) ${ }^{4}$. Con la llegada de la Segunda Guerra Mundial, Wood ingresa en el Servicio de Emergencias Médicas y en 1942 se incorpora como oficial en el Cuerpo Médico del Real Ejército Británico siendo designado encargado de los Hospitales Militares de la campaña del Norte de África e Italia. Alcanza el grado de Brigadier en 1945 y por sus servicios es condecorado con la Orden del Imperio Británico (OBE). Es dado de baja en 1946 y se inicia en práctica privada, pero al poco tiempo Sir Francis Fraser (1885-1964) lo invita a volver a Hammersmith para organizar el Instituto de Cardiología donde realizó sus primeros cateterismos cardiacos. Esto le produjo gran placer al poder realizar mediciones hemodinámicas y correlacionar las mismas con los hallazgos clínicos. Es nombrado Decano y Director del Instituto y del National Heart Hospital, y Médico a Cargo del Departamento de Cardiología del Brompton Hospital. Para un médico de sólo 40 años estas distinciones sorprendieron a la comunidad médica de su tiempo, pero rápidamente quedó demostrado que eran bien merecidas ${ }^{2,4}$. 


\section{Su libro}

Aquí comienza el período más fructífero de su vida, donde combina sus cualidades de clínico minucioso con la información proveniente del laboratorio de cateterismo. Durante esta época ya había iniciado un borrador de su magistral libro de texto "Enfermedades del Corazón y de la Circulación" cuya primera edición se publicó en Londres en $1950^{5}$. Esta obra está basada principalmente en su experiencia personal, enriquecida por la experiencia lograda en el laboratorio de cateterismo y en el quirófano del cardiocirujano Sir Russell Brock (1903-1980) donde pudo plasmar la integración de la clínica y la fisiopatología con la anatomía ${ }^{4}$. A diferencia con la actualidad en que el investigador clínico envía a publicar sus trabajos a revistas con arbitraje de sus pares, la mayor publicación de Wood fue su libro. Esta obra le trajo el reconocimiento de todo el mundo como la autoridad europea más relevante de la cardiología del momento. La segunda edición apareció en 1956 y fue traducida a varios idiomas; siendo el traductor de la versión española M. Torner Soler de la Escuela de Cardiología de la Universidad de Barcelona ${ }^{6}$. Wood estaba preparando la tercera edición cuando lo sorprendió la muerte. Un grupo de sus colaboradores y discípulos encabezados por Walter Somerville (1937-2005) decide completar la obra, que se publica en 1968, seis años después de su muerte ${ }^{7,8}$. Publicó además casi una centena de artículos en revistas indexadas.

\section{Sus intereses, aportes y personalidad}

Sus mayores intereses lo constituyeron las cardiopatías congénitas ${ }^{9-21}$, las valvulopatías reumáticas $^{10,15-17,19}$ y la hipertensión pulmonar ${ }^{9,13,21}$. Su experiencia en cardiopatías congénitas fue reflejada en una cuantiosa serie de más de 1000 casos clínicos con diagnósticos sustentados mediante cateterismo cardiaco y en muchos casos con confirmación intra-operatoria o anatomopatológica $^{11,12}$. Llamó la atención sobre la existencia de casos de tetralogía de Fallot acianóticos en reposo, con saturaciones de oxígeno arterial entre 87 y $97 \%{ }^{14}$, y de casos hipercianóticos con síncope ${ }^{20}$. Supo hacer la distinción entre el soplo de Carey Coombs de la carditis reumática aguda y el soplo diastólico mitral de las comunicaciones interventriculares con gran hiperflujo transvalvular mitral, un diagnóstico diferencial de mucha im- portancia en su época ${ }^{14}$. Redefinió al "complejo de Eisenmenger" como una situación fisiopatológica esencialmente debida a hipertensión pulmonar con shunt invertido o bidireccional, restándole importancia a la localización anatómica del shunt ${ }^{18}$. Fue el propulsor de expresar la resistencia vascular pulmonar en simples unidades de resistencia (Unidades Wood) tomando los datos de la diferencia de presiones en $\mathrm{mmHg}$ y el gasto pulmonar en litros por minuto (multiplicando las unidades por 80 se convierten en dinas-seg- $\mathrm{cm}^{-5}$ ). Hizo suya la hipótesis que en ciertas condiciones la misma hipertensión pulmonar es capaz de generar vasoconstricción arteriolar pulmonar y que este mecanismo ulteriormente podría ser el responsable de perpetuar el proceso, aun en casos en que la causa inicial ya hubiera desaparecido. En el caso de la estenosis mitral, puso en evidencia que la vasoconstricción arteriolar pulmonar intervenía en el mecanismo de la hipertensión pulmonar y que el efecto podía revertirse con la inyección de acetilcolina en la arteria pulmonar. Explicó como esta situación de vasoconstricción proximal a los capilares pulmonares actuaría como barrera protectora contra el edema agudo de pulmón, a expensas de una reducción del gasto cardiaco y del eventual desarrollo de insuficiencia cardiaca derecha ${ }^{9,13,15-17,19,21}$.

No vaciló en utilizar métodos no convencionales de enseñanza llegando a realizar una filmación para ejemplificar las distintas características del pulso venoso yugular ${ }^{2}$. Se caracterizó por su estilo intensamente honesto: los errores jamás se ocultaban, más bien los visualizaba como un desafío que requería una clara identificación y comprensión de sus causas, para que no se repitieran ${ }^{1}$. En la cabecera del paciente, delante de sus discípulos y estudiantes, se lo reconocía por su aguda personalidad y su exquisita habilidad diagnóstica. Tenía grandes dotes histriónicas y era capaz de envolver a su audiencia, pero simultáneamente podía ser cáustico y agresivo en sus comentarios. Si bien muchos se sentían estimulados por su estilo argumentativo, hubo personalidades de la época que se sintieron ofendidos. Sus comentarios más mordaces generalmente eran dirigidos a sus colegas mayores que no estaban acostumbrados a que sus diagnósticos y opiniones fueran cuestionados, en especial por un profesional más joven. Todos los tradicionales conceptos cayeron bajo su lupa, mientras intentaba siempre fundamentar sus diag- 
Paul Wood - J.D. Humphreys et al

nósticos y tratamientos en hechos fisiopatológicos comprobables. De esta manera se adelanta en el tiempo y se convierte en un precursor del ejercicio de la medicina basada en evidencia. Durante la década 1950-1960 el National Heart Hospital de Londres se convirtió en un destino preferido de estudiosos de la cardiología de todo el mundo y Wood era la estrella indiscutida del "show". Fuera de la práctica médica sus amigos se deleitaban con el anfitrión agradable y cordial, que tenía un agudo sentido del humor. Su hobby más allá de la cardiología fue la jardinería ${ }^{2}$.

Tal vez las exigencias del ejercicio profesional, la tarea intensa de preparar la tercera edición de su libro e indudablemente el hecho de que era un gran fumador de cigarrillos, hicieron que a la edad de 54 años, estando en su consultorio tuvo un infarto de miocardio. Fue internado el Middlesex Hospital de Londres e instruyó a los médicos que lo asistían que ante la eventualidad de un paro cardiaco, no debían intentar ninguna medida de resucitación, “o volverépara espantarlos". Dos días después, el 13 de julio de 1962 tuvo un paro cardiaco y falleción ${ }^{2,7}$.

Nada mejor que las palabras de Wood en los últimos dos párrafos del prefacio de la primera edición de su libro para definir su personalidad y pensamiento: "Los clínicos más conservadores que han sido testigos de este desarrollo tecnológico (se refiere a los avances de la cardiología durante la primera mitad del siglo XX) pueden haberlo recibido con algún recelo, pero son relativamente pocos los que han expresado puntos de vista reaccionarios. Sin embargo, ya hay bastante evidencia que muestra que estamos en peligro de perder nuestro legado clínico, y depositar demasiada confianza en cifras registradas por máquinas. La Medicina sufrirá si esta tendencia no se controla.

Al presentar este libro, he intentado mantener el apropiado equilibrio entre el hombre y los instrumentos, entre la experiencia y las estadísticas, entre lo tradicional y lo heterodoxo, entre la medicina junto a la cabecera del enfermo y las pruebas especiales, entre la medicina práctica y la académica; y de esta manera enlazar el pasado con el presente"s.

\section{Referencias}

1. Katz AM. Icons of Cardiology. Paul Hamilton Wood: Clinician-Scientist. Dialogues Cardiovasc Med 2004; 9: 117-20.

2. Dimond EG. The Meteoric Career of Paul Wood. En:
Hurst JW, Conti CR, Fye WB, Editores, Profiles in Cardiology. United States: Editorial Foundation for Advances in Medicine and Science Inc.; 2003. p 334-335.

3. Silverman ME, Somerville W. To die in one's prime: the story of Paul Wood. Am J Cardiol 2000; 85: 75-88.

4. Bauer G. Wood, Paul Hamilton (1907-1962). En: Australian Dictionary of Biography Editores, Volume 16. Melbourne, Australia: Melbourne University Press.; 2002. p 579-580.

5. Wood P. Diseases of the Heart and Circulation. London. Editorial Eyre \& Spottiswoode.; 1950.

6. Wood P. Enfermedades del Corazón y de la Circulación. Barcelona. Editorial Toray S.A.; 1962.

7. Sommerville W. Obituary. Paul Wood. BMJ 1962; 2: 262-3.

8. Paul Wood's Diseases of the Heart and Circulation. Revised and Enlarged by his Friends and Colleagues. London. Editorial Eyre \& Spottiswoode.; 1968.

9. Wood P. Electrocardiographic appearances in acute and chronic pulmonary heart disease. Br Heart J 1948; 10: 87.

10. Wood P. Discussion on the management of rheumatic fever and its early complications; cardiac complications. Proc Roy Soc Med 1950; 43: 195-9.

11. Wood P. Congenital heart disease; a review of its clinical aspects in the light of experience gained by means of modern techniques. I. BMJ 1950; 2: 639-45.

12. Wood P. Congenital heart disease; a review of its clinical aspects in the light of experience gained by means of modern techniques. II. BMJ 1950; 2: 693-8.

13. Wood P. Pulmonary hypertension. Br Med Bull 1952; 8: 348-43.

14. Wood P, Magidson O, Wilson PA. Ventricular septal defect with a note on acyanotic Fallot's tetralogy. Br Heart J 1954; 16: 387-406.

15. Wood P. An appreciation of mitral stenosis. BMJ 1954; 1: 1051-63.

16. Wood P. An appreciation of mitral stenosis. BMJ 1954; 1: 1113-24.

17. Wood P, Besterman EMM, Towers MK, McIlroy MB. The effect of acetilcholine on pulmonary vascular resistance and left atrial pressure in mitral stenosis. Br Heart J 1957; 19: 279-86.

18. Wood P. The Eisenmenger syndrome or pulmonary hypertension with reversed central shunt. I. BMJ 1958; 2: 701-9.

19. Wood P. Aortic stenosis. Am J Cardiol 1958; 1: 553-71.

20. Wood P. Attacks of deeper cyanosis and loss of consciousness (syncope) in Fallot's tetralogy. Br Heart J 1958; 20: 282-6.

21. Davis H, Williams J, Wood P. Lung stiffness in states of abnormal pulmonary blood flow and pressure. Brompton Hosp Rep 1962; 31: 235-47. 